\title{
Persistent Neutrophil Dysfunction and Suppression of Acute Lung Injury in Mice following Cecal Ligation and Puncture Sepsis
}

\author{
Jamison J. Grailer Miriam Kalbitz Firas S. Zetoune Peter A. Ward \\ Department of Pathology, University of Michigan Medical School, Ann Arbor, Mich., USA
}

\author{
Key Words \\ Neutrophil · Macrophage · Innate immune suppression . \\ Lung inflammation
}

\begin{abstract}
Sepsis, both in humans and in rodents, is associated with persistent immunosuppression accompanied by defects in innate immunity during the acute phase of sepsis. Mice were rendered septic by cecal ligation and puncture (CLP) followed by the induction of acute lung injury, employing distal airway deposition of $\mathrm{IgG}$ immune complexes, in order to quantitatively evaluate innate immune responses following the induction of sepsis. Suppression of innate immune responses in the lung occurred as early as $12 \mathrm{~h}$ after CLP and up to 21 days thereafter. The mechanism of innate immune defects included a reduced leak of albumin into the lungs together with reduced levels of tumor necrosis factor in bronchoalveolar lavage fluids and increased levels of interleukin-10 that were persistent. Bone marrow-derived neutrophils (polymorphonuclear neutrophils; PMNs) from CLP mice also had reduced levels of the activation marker CD11 b and a depressed respiratory burst following stimulation in vitro. These results were not observed in mice with endotoxemia, where the innate inflammatory response was preserved. However, sustained lymphopenia was present in both models, suggesting differential regulation of innate and adaptive immunity in the two sepsis models. These data
\end{abstract}

indicate that CLP induced a prolonged suppression of inflammatory responses both in the lung and systemically, as defined by bone marrow-derived PMN dysfunction.

๑) 2014 S. Karger AG, Basel

\section{Introduction}

Sepsis is a life-threatening illness resulting in significant morbidity and an estimated mortality of $20-40 \%$ [1]. Early studies have suggested that the mortality associated with sepsis is due primarily to the robust activation of leukocytes, the overproduction of inflammatory mediators, and the resulting tissue damage and multiorgan failure (i.e. the cytokine storm) [2]. However, therapeutics targeting the inflammatory response have not resulted in significant survival benefits. There are currently no FDA-approved drugs to improve the clinical outcome of sepsis. Due to improvements in supportive care, most patients now survive the acute septic episode. However, mortality is frequently attributed to subsequent opportunistic infections, often of nosocomial origin [3]. Survivors of sepsis show an increased mortality and a reduced quality of life weeks, months, and years after resolution of the acute condition [4]. The etiology of these long-term effects remains unknown, and there are numerous infectious and noninfectious conditions that are associated with a decreased life expectancy after

\section{KARGER}

(c) 2014 S. Karger AG, Basel

1662-811X/14/0065-0695\$39.50/0

E-Mail karger@karger.com

www.karger.com/jin
Dr. Peter A. Ward

Department of Pathology, University of Michigan Medical School

1301 Catherine Rd.

Ann Arbor, MI 48109 (USA)

E-Mail pward@med.umich.edu 
'recovery' from sepsis. Opportunistic infections (often respiratory) are frequently found in survivors of sepsis [5], which would suggest a sepsis-induced immune system dysfunction. Indeed, rodent models of Aspergillus fumigatus and Pseudomonas aeruginosa respiratory infection following cecal ligation and puncture (CLP) sepsis have showed that mice were at a higher risk for persistent infection and death compared to nonseptic mice [5-7]. Our lab has described polymorphonuclear neutrophil (PMN) dysfunction during sepsis, including defects in cytokine production and the respiratory burst which were dependent on the complement activation product C5a [8-10]. Furthermore, reports have indicated that sepsis alters myelopoiesis [11], reduces monocyte HLA-DR expression and antigen presentation $[12,13]$, reduces proinflammatory cytokine production [14], and enhances anti-inflammatory interleukin (IL)-10 production [14]. However, these reports have focused on time points relatively early (up to $24 \mathrm{~h}$ ) after the induction of sepsis. It is not clear whether these defects are sustained in the days and weeks following sepsis.

Acute lung injury (ALI) is characterized by the production of proinflammatory mediators such as complement activation products, cytokines, and chemokines and the accumulation of PMNs in the lung. Disruption of the blood-alveolar barrier due to vascular endothelial and alveolar epithelial cell damage/death, in concert with a reduced ability to clear alveolar fluid, results in pulmonary edema and lung consolidation, intrapulmonary hemorrhage, and a severely impaired gas exchange [reviewed in 15]. The experimental model of ALI generated by the distal airway deposition of IgG immune complexes (IC) is primarily dependent on the innate immune response, characterized by neutrophilic alveolitis, and mimics many parameters of human ALI [reviewed in 16].

In this report, we develop a 'two-hit' model of lowgrade CLP-induced sepsis (20\% mortality after 7 days) followed by the induction of ALI after airway deposition of IgG IC in order to investigate prolonged sepsis-induced suppression of the innate inflammatory response in the lung. This model has several advantages for investigating innate inflammatory responses following CLP. First, low-grade CLP reflects the survival rate of the human sepsis. Second, the use of an IgG IC ALI model in lieu of microbial products [e.g. lipopolysaccharides (LPS)] avoids complications of data interpretation arising from issues such as Toll-like receptor tolerance induced by the first 'hit'. Finally, the use of IgG IC instead of infectious agents allows direct investigation of localized lung inflammation since there is no systemic dissemination of pathogens causing widespread cell activation. Using this model, we describe prolonged suppression of the innate inflammatory response in the lung following CLP, which includes alterations in both PMN and alveolar macrophage function. This phenomenon was not observed in endotoxemic mice.

\section{Materials and Methods}

\section{Animals}

All procedures were performed in accordance with US National Institutes of Health guidelines and were approved by the University of Michigan Committee on the Use and Care of Animals. Male, age-matched (8-9 weeks old) C57BL/6 mice were purchased from the Jackson Laboratories (Bar Harbor, Me., USA). RAG-1 $1^{-1-}$ mice were on the C57BL/6 background (Jackson Laboratories). All animals were housed under specific pathogen-free conditions with free access to food and water.

\section{CLP and Endotoxemia}

Low-grade CLP was used for this study, as described previously [17]. Briefly, following anesthesia with ketamine and xylazine, the cecum was exposed, ligated (5-7 $\mathrm{mm})$, and punctured through and through using a 21-gauge needle. A small amount of intestinal content was extruded to ensure the patency of the puncture. The cecum was repositioned and the abdominal cavity was closed in layers with simple stiches using Prolene 5-0 (Ethicon, Somerville, N.J., USA). Sham animals underwent the same procedure without ligation and puncture. Mice were rendered endotoxemic by i.p. administration of LPS (Escherichia coli o111:B4; Sigma, St. Louis, Mo., USA) at $10 \mathrm{mg} / \mathrm{kg}$ of body weight.

\section{Acute Lung Injury}

IgG IC ALI was performed as previously described [16]. Briefly, following anesthesia with ketamine and xylazine, mice received $125 \mu \mathrm{g}$ rabbit anti-bovine serum albumin antibody (MP Biomedicals, Solon, Ohio, USA) during inspiration in a volume of $30 \mu \mathrm{l}$ saline. Then, $1 \mathrm{mg}$ BSA (Sigma) was injected i.v. in $200 \mu \mathrm{l}$ saline (sham mice received saline only i.v.). Six hours later, BALF was harvested by the slow instillation and retraction of $1 \mathrm{ml}$ PBS. BALF leukocytes were enumerated on a hemocytometer following lysis of erythrocytes. Leukocyte differentials were determined by cytospin preparations stained with Wright-Giemsa (Thermo Fisher, Waltham, Mass., USA). For each sample, more than 200 cells were analyzed from random fields. The BALF was aliquoted and stored at $-80^{\circ} \mathrm{C}$ until use. A useful gauge of inflammatory tissue damage during ALI is the leak of albumin from the blood into the alveolar compartment (indicating alveolar epithelial and endothelial barrier breakdown) [18]. Therefore, we used BALF albumin levels as indicators of inflammatory injury.

\section{Blood Collection}

Heparinized blood was collected from the retro-orbital venous plexus following anesthesia with isoflurane. Total nucleated cell (leukocyte) counts were determined on a hemocytometer following lysis of erythrocytes with $2 \%$ acetic acid. Leukocyte dif- 
ferential counts were determined with blood smears stained with Wright-Giemsa. More than 200 cells were analyzed per blood sample.

\section{PMN Isolation}

Mouse PMNs were harvested from bone marrow by flushing bilateral femurs with Hank's balanced salt solution (HBSS). Erythrocytes were lysed in hypotonic buffer. Cells were washed in HBSS and layered on Histopaque 1077 (Sigma) for density gradient centrifugation $\left(500 \mathrm{~g}, 30 \mathrm{~min}, 4^{\circ} \mathrm{C}\right)$. The pellet (PMNs) was washed with HBSS prior to downstream applications. The PMN purity was $85 \pm 3 \%$ (mean $\pm \mathrm{SD}$ ) and $82 \pm 1 \%$ as determined by flow cytometry $\left(\mathrm{Ly} 6 \mathrm{G}^{+} \mathrm{CD} 11 \mathrm{~b}^{+}\right)$and Wright-stained cytospin preparations, respectively.

\section{In vitro Assays and Flow Cytometry}

PMNs were exposed to recombinant mouse C5a (R\&D Systems, Minneapolis, Minn., USA) or phorbol 12-myristate 13-acetate (PMA; Sigma) for $1 \mathrm{~h}$ at $37^{\circ} \mathrm{C}$. Cells were washed and labeled for flow cytometric analysis on a BD LSR-II flow cytometer equipped with FACSDiva software (both from BD Biosciences, San Jose, Calif., USA). Data was analyzed using FlowJo software (TreeStar, Ashland, Oreg., USA). PerCP-Cy5.5-conjugated antiLy6G (BD Biosciences), PE/Cy7-conjugated anti-CD11b (eBioscience, San Diego, Calif., USA), PE/Cy7-conjugated anti-C5aR (eBioscience), purified anti-gp91 $1^{\text {phox }}$ (BD Biosciences), and FITCconjugated anti-mouse IgG (Jackson Immunoresearch) were used for this study. Irrelevant isotype control antibodies were used to determine the levels of nonspecific labeling. Cells with the forward and side scatter properties of PMNs were further gated on

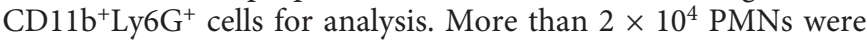
analyzed from each sample.

The reactive oxygen species ( $\mathrm{ROS} ; \mathrm{O}_{2}^{-}$) production was determined by the superoxide dismutase (SOD)-inhibitable reduction of ferricytochrome $c$, as described previously [8]. Briefly, PMNs $\left(5 \times 10^{5}\right)$ were incubated in HBSS with ferricytochrome $c(80 \mu \mathrm{M}) \pm$ SOD $(30 \mathrm{U} / \mathrm{ml})$ in the presence or absence of C5a or PMA for $1 \mathrm{~h}$ at $37^{\circ} \mathrm{C}$. After centrifugation, supernatants were transferred to $96-$ well plates and the absorbance $(550 \mathrm{~nm})$ was determined on a microplate reader. The amount of $\mathrm{O}_{2}^{-}$production was calculated based on the molar amount of reduced ferricytochrome $c$ in the absence or presence of SOD. The amount of $\mathrm{O}^{-}{ }_{2}$ generated was normalized to the percentage of PMNs in the cell preparation. Reagents for these assays were from Sigma.

\section{ELISA}

Cytokine ELISAs were from R\&D Systems, and mouse albumin ELISAs were from Bethyl Laboratories (Montgomery, Tex., USA). ELISAs were performed as per the manufacturer's recommendations. Mouse albumin ELISAs do not cross-react with BSA.

\section{Statistical Analysis}

Data were expressed as means \pm SEM. Data were analyzed using GraphPad Prism 6 graphing and statistical analysis software (GraphPad Inc., La Jolla, Calif., USA). Significant differences between groups were determined by one-way ANOVA followed by Dunnett's multiple comparisons tests. Where appropriate, significant differences between individual sample means were determined using Student's t test. $\mathrm{p}<0.05$ was considered statistically significant.

Prolonged CLP-Induced Innate Immune Suppression

\section{Results}

Prolonged Suppression of IgG IC-Induced Lung Injury following CLP

We determined whether CLP alone would induce a lung inflammatory response in mice. The albumin levels (a measure of epithelial/endothelial barrier breakdown) as well as the PMN numbers in BALF were not significantly increased at any time point following CLP (fig. 1a). At 0.5 and $1 \mathrm{~h}$ after CLP, the PMN numbers appeared to be reduced, although this did not reach statistical significance (ANOVA, $p=0.10$ ). These data suggest that, in this CLP model, the albumin leak into the lung in both the non-CLP and the CLP groups was minimal. This is in contrast to some reports describing lung inflammation following CLP in mice $[19,20]$.

To investigate the long-term effects of sepsis on the innate immune response in the lung, we developed a twohit model involving CLP-induced sepsis followed by IgG IC ALI at various time points after CLP. Twelve hours to 21 days after CLP, ALI was induced by alveolar deposition of IgG IC, and BALFs were harvested $6 \mathrm{~h}$ later (fig. 1b). Using this model, we measured parameters of the innate inflammatory response at the indicated time points following the induction of sepsis.

To demonstrate that the IgG IC ALI model was primarily dependent on the innate response, IgG IC-induced lung inflammation was compared in wild-type mice and RAG- $1^{-/}$mice, which lack mature $\mathrm{T}$ and $\mathrm{B}$ cells. The results showed that the albumin levels in BALF were not significantly reduced in the RAG-1 ${ }^{-/-}$mice following IgG IC deposition (fig. 1c). Furthermore, the number of PMNs found in BALF was similar between wild-type and RAG-1 $1^{-/-}$mice (wild type: $12 \pm 1.9 \times 10^{4} \mathrm{vs}$. RAG-1 ${ }^{-/}: 9 \pm$ $\left.0.3 \times 10^{4}, \mathrm{p}=0.21\right)$. Therefore, the IgG IC ALI model is primarily dependent on the innate inflammatory response involving PMNs and macrophages.

During IgG IC-induced ALI, the albumin levels found in BALF were significantly elevated compared to those in BALF from sham ALI animals (fig. $1 \mathrm{~d}$, as shown in the sham ALI and non-CLP groups, $\mathrm{p}<0.005$ ). When mice underwent IgG IC ALI following CLP, BALF albumin levels were significantly attenuated at all but one time point after CLP (day 7) compared to BALF from the nonCLP group (fig. 1d). Early time points ( $\leq 3$ days) after CLP showed the largest reduction in BALF albumin levels (70$90 \%$ decrease compared to the non-CLP group; fig. 1d). The BALF albumin levels modestly increased at later times point but were still significantly reduced compared to the non-CLP group up to 21 days after CLP (fig. 1d). 


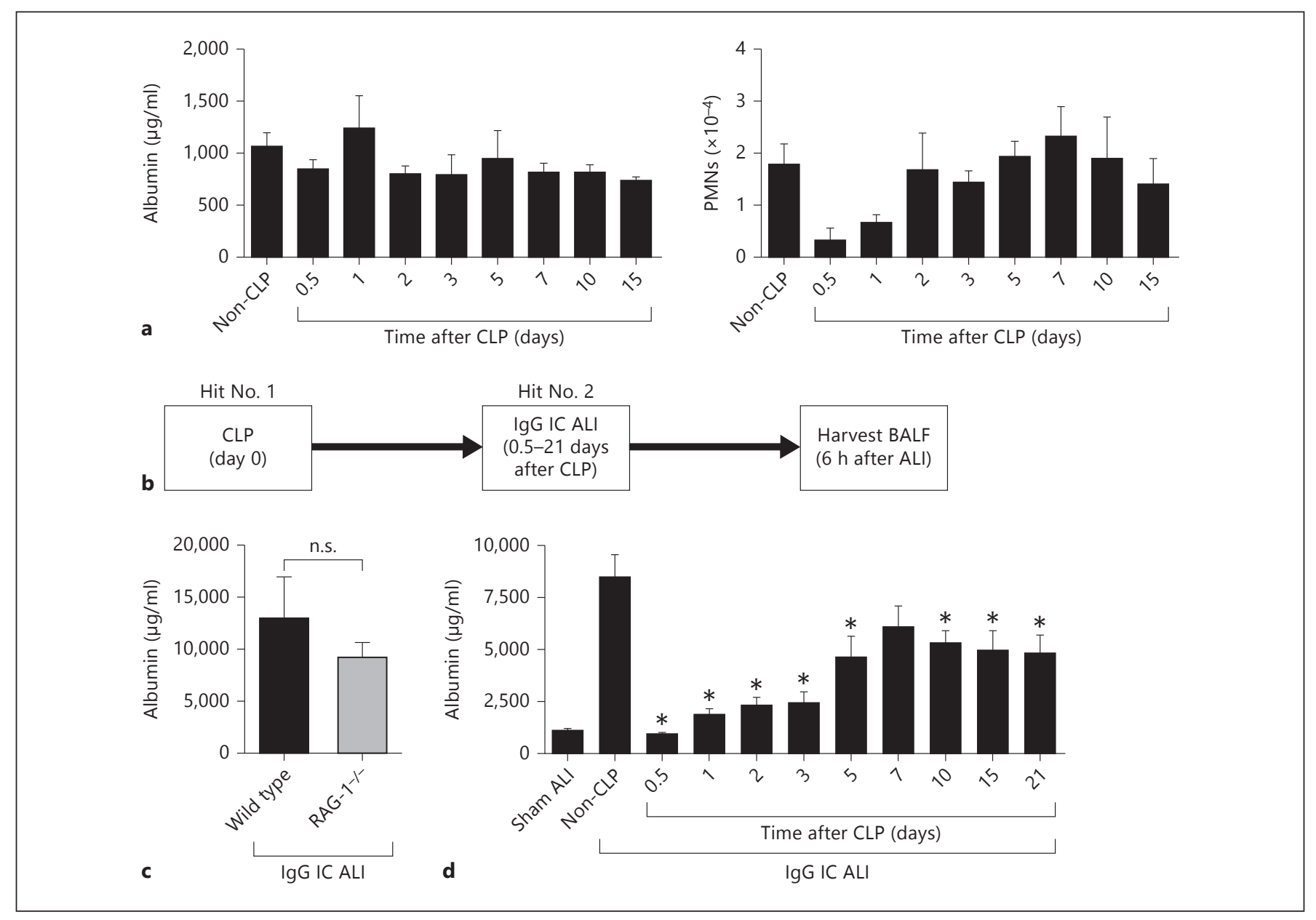

Fig. 1. Prolonged lung inflammatory suppression following CLPinduced sepsis. a Levels of albumin and PMN numbers in BALF at different time points following CLP ( $\mathrm{n} \geq 4$ mice per group). b A two-hit model of CLP sepsis followed by IgG IC ALI was used. At different time points following CLP, IgG IC were deposited in the distal airway and BALF was harvested $6 \mathrm{~h}$ later. $\mathrm{c}$ Levels of albumin

Therefore, following CLP, there was a prolonged (at least 21 days) suppression of the lung innate inflammatory response based on the albumin leak into the lung.

\section{CLP-Induced Alterations in the Lung and Circulating PMN Numbers}

PMN accumulation in the lung is a hallmark of ALI. In the IgG IC ALI model, we observed a significant increase in PMNs in BALF compared to BALF from sham mice (fig. 2a, compare the sham ALI and non-CLP groups, $\mathrm{p}<$ 0.005). CLP affected lung PMN recruitment during IgG IC ALI in a biphasic manner. Specifically, the PMN numbers in BALF from ALI mice were significantly reduced at early ( $\leq 24 \mathrm{~h}$ ) time points following CLP (fig. 2a). However, found in BALF following IgG IC ALI in wild-type and RAG-1-1mice $(\mathrm{n}=5$ mice per group). n.s. $=$ Not significant. $\mathbf{d}$ Albumin levels in BALF following CLP and IgG IC ALI ( $\mathrm{n} \geq 5$ mice per group). ${ }^{*}$ Differences between the non-CLP and CLP groups were significant, $\mathrm{p}<0.05$.

the BALF PMN numbers recovered at subsequent time points and were significantly increased 7 days after CLP. The increased numbers of PMNs in the lung may explain why inflammatory suppression was lost on day 7 after CLP (fig. 1d). In addition, such an increase in PMN numbers may mask the impact of CLP-induced PMN functional defects (described in subsequent figures) on ALI. The PMN numbers in BALF returned to non-CLP levels after day 7 (fig. 2a). Therefore, CLP significantly altered the PMN accumulation in the lung during IgG IC ALI.

Blood PMN numbers were determined at different time points following CLP to compare the number of circulating PMNs to the number of lung PMNs. IgG IC ALI did not significantly alter the numbers of blood PMNs af- 


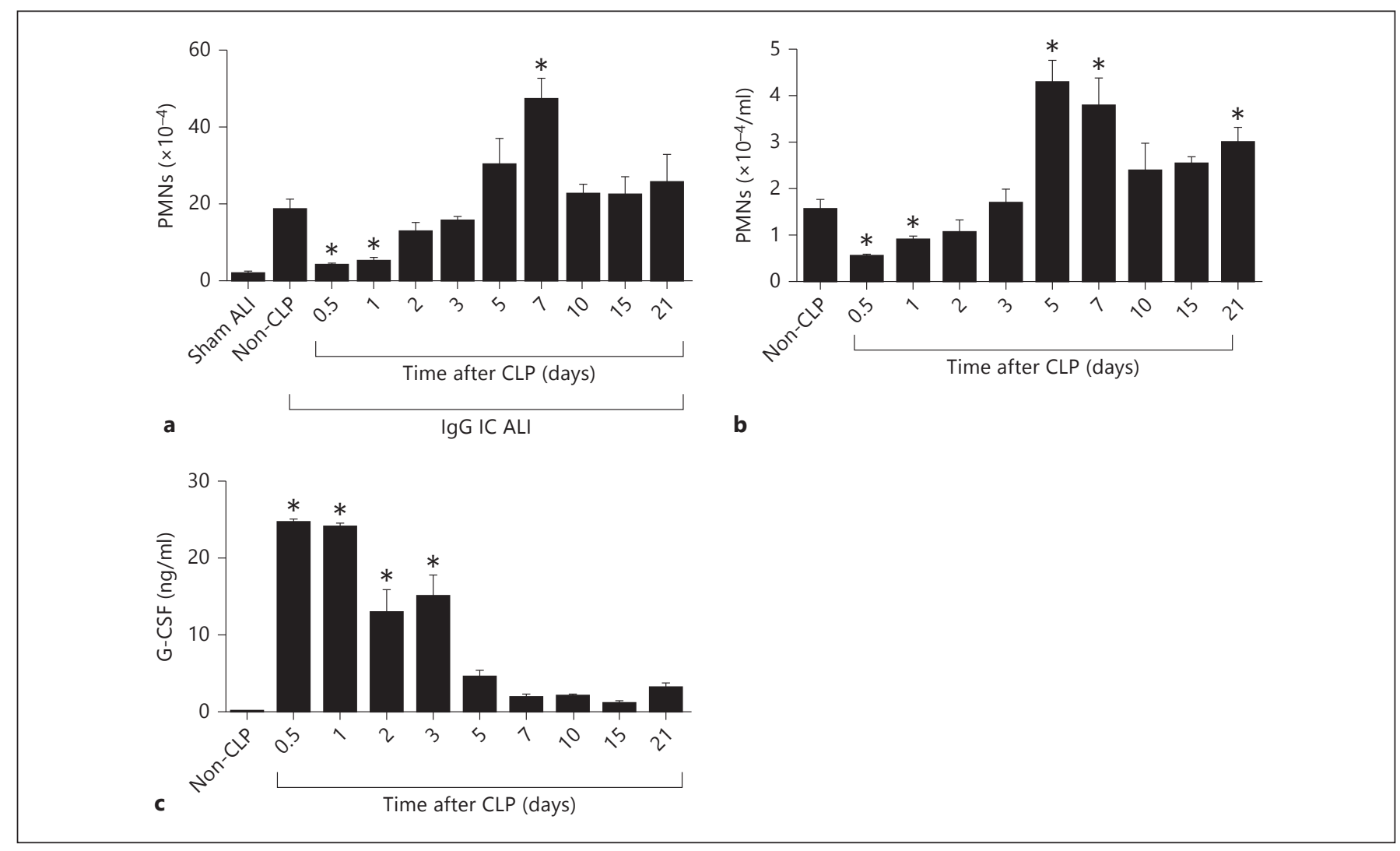

Fig. 2. CLP-induced alterations in PMN numbers. a Numbers of PMNs found in BALF during the two-hit model of CLP and IgG IC ALI at different time points following CLP, as described in figure 1 ( $n \geq 5$ mice per group). $\mathbf{b}$ Number of blood PMNs at different time points following CLP ( $\mathrm{n} \geq 5$ mice per group). c Levels of GCSF in plasma at different time points following CLP, as determined by ELISA ( $\mathrm{n} \geq 5$ mice per group). ${ }^{*}$ Differences between the non-CLP and CLP groups were significant, $\mathrm{p}<0.05$. ter $6 \mathrm{~h}\left[1.6 \pm 0.2 \times 10^{6} / \mathrm{ml}\right.$ vs. $1.1 \pm 0.3 \times 10^{6} / \mathrm{ml}$ for before and after ALI $(6 \mathrm{~h})$, respectively; $\mathrm{p}=0.25$ ]. The numbers of blood PMNs were significantly reduced at early $(\leq 24 \mathrm{~h})$ time points following CLP (fig. 2b). Blood PMN numbers were significantly increased compared to non-CLP numbers at 5-7 days following CLP and PMN numbers remained elevated 21 days after CLP, although the day 10 and day 15 time points did not reach statistical significance (fig. 2b). Importantly, the number of BALF PMNs was positively correlated with the number of blood PMNs at different time points after CLP $(\mathrm{p}<0.0005, \mathrm{r}=0.92)$. The levels of CXC chemokines in BALF from ALI mice were reduced by CLP at the 24-hour time point (by 52 and $51 \%$ for KC and MIP-2, respectively). However, the CXC chemokine expression was restored by day 3 after CLP (values in $\mathrm{pg} / \mathrm{ml}$ for non-CLP and day 3 CLP, respectively: KC: $5,466 \pm 1,015$ vs. $6,186 \pm 521, \mathrm{p}=0.49$; MIP-2: 4,291 \pm 340 vs. $4,480 \pm 693, \mathrm{p}=0.79)$. Therefore, the reduced PMN accumulation during IgG IC ALI at early $(\leq 24 \mathrm{~h})$ time points following CLP may have been the result of the reduced availability of circulating PMNs or the reduced production of CXC chemokines during IgG IC ALI.

Granulocyte colony-stimulating factor (G-CSF) is a cytokine that stimulates granulopoiesis during homeostasis and during infection [21, 22]. Elevated levels of G-CSF may explain the surge in PMNs we observed after CLP (day 5-7; fig. 2b). Therefore, we measured the levels of GCSF in plasma at different time points after CLP. The results showed that G-CSF was elevated up to 3 days after CLP; the levels were reduced thereafter (fig. 2c). Thus, GCSF likely has a role in priming granulopoiesis after CLP, as has been described in mice during endotoxemia [23].

\section{Prolonged Dysfunction of PMNs after CLP}

While a significant reduction in BALF and blood PMNs was apparent at early time points after CLP, it does not explain the suppression of albumin leakage into the lung at later time points ( $>2$ days after CLP) when circulating PMN 


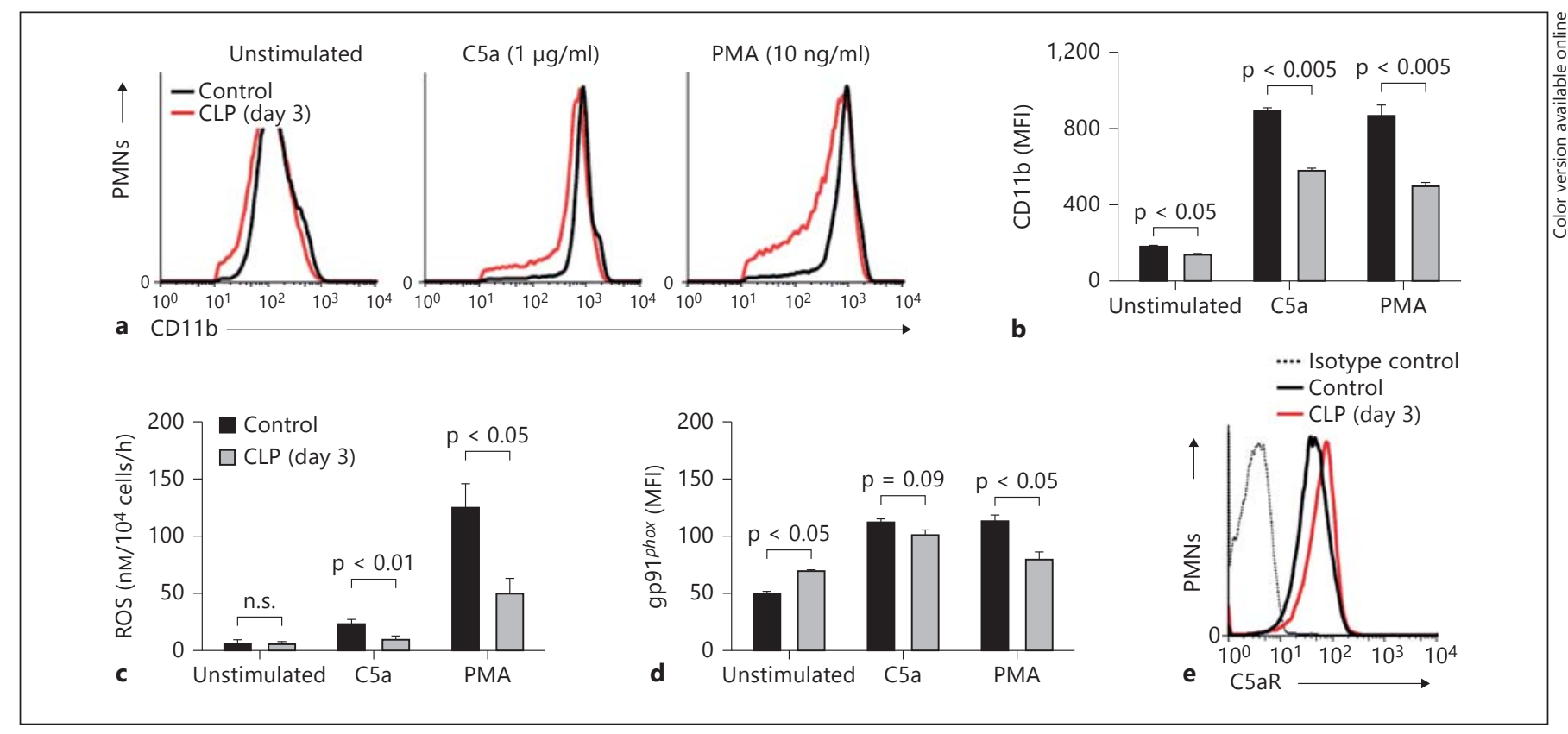

Fig. 3. Prolonged PMN dysfunction induced by CLP. a-d Bone marrow PMNs isolated from control or CLP (day 3 ) mice were incubated in the presence or absence of C5a $(1 \mu \mathrm{g} / \mathrm{ml})$ or PMA $(10$ $\mathrm{ng} / \mathrm{ml}$ ) for $1 \mathrm{~h}$. a Surface CD11b levels. Plots are representative of 5 mice per group. b Aggregate mean fluorescence intensity (MFI)

numbers and lung PMN accumulation were restored and even enhanced above basal levels. We hypothesized that CLP induced PMN dysfunction at these later time points, leading to reduced inflammation and tissue damage. We chose a time point to evaluate PMN activation and the generation of ROS after CLP (3 days) in which BALF and blood PMN numbers were within the normal range (fig. 2) but in which there were still significant reductions in ALI-induced albumin leakage into the lung (fig. 1d). Bone marrow-derived PMNs from control (sham CLP) or CLP (day 3) mice were activated in vitro with complement anaphylatoxin C5a $(1 \mu \mathrm{g} / \mathrm{ml})$ or with PMA $(10 \mathrm{ng} / \mathrm{ml})$. Surface expression of the PMN activation marker CD11b was analyzed by flow cytometry. For unstimulated cells, CD11b expression was 20\% lower on CLP PMNs compared to control (sham) PMNs [fig. 3a (left), b (unstimulated)]. Both C5a and PMA significantly elevated the cell surface CD11b expression on both control and CLP PMNs, indicating that PMN activation occurred. However, the CD11b expression on CLP PMNs in response to C5a or PMA was reduced by 35 and $43 \%$, respectively, compared to control (sham) PMNs (fig. 3a, b). Therefore, CLP impaired PMN CD11b expression in response to $\mathrm{C} 5 \mathrm{a}$ or PMA, indicating a defect in PMN responsiveness. Alternatively, these data may re- from the experiments in $\mathbf{a}$ ( $\mathrm{n}=5$ mice per group). $\mathbf{c}$ SOD-inhibitable $\mathrm{O}_{2}{ }^{-}$generation $(\mathrm{n}=5$ mice per group). n.s. $=$ Not significant. d Surface gp91 ${ }^{\text {phox }}$ expression ( $\mathrm{n}=5$ mice per group). e Surface expression of $\mathrm{C} 5 \mathrm{aR}$ on bone marrow PMNs from control or CLP (day 3) mice. The plot is representative of 4 mice per group.

flect the appearance of a CD11 bow population of PMNs in bone marrow following CLP sepsis.

ROS is a prominent myeloid cell-derived inflammatory product during ALI. Activated PMNs, in particular, can generate large amounts of ROS which produce defects in lung epithelial/endothelial barrier function through direct cell damage and the activation of signaling pathways resulting in the disruption of cell junctional proteins and the formation of intercellular gaps [24, 25]. We analyzed ROS $\left(\mathrm{O}_{2}^{-}\right)$generation in C5a- or PMAstimulated control or CLP PMNs (obtained 3 days after CLP). Both C5a and PMA induced ROS generation by control PMNs $(p<0.05)$. However, in response to C5a or PMA, CLP PMNs generated 58 and 60\% less ROS, respectively, compared to control PMNs (fig. 3c). Therefore, a prolonged defect in ROS generation occurred in bone marrow PMNs following CLP.

ROS generation by PMNs is mediated by nicotinamide adenine dinucleotide phosphate (NADPH) oxidase, which consists of a membrane-bound subunit, flavocytochrome b558 (gp91 ${ }^{\text {phox }}$ and p22 $2^{\text {phox }}$ subunits), and the cytosolic components $\mathrm{p} 47^{\text {phox }}, \mathrm{p} 40^{\text {phox }}, \mathrm{p} 67^{\text {phox }}$, and Rac2. In unstimulated PMNs, only $10-20 \%$ of the total flavocytochrome b558 is expressed on the cell surface, while most of 
it resides in the membrane components of secretory vesicles and granules. PMN activation causes the fusion of vesicles and granules with the plasma membrane, resulting in an increased surface expression of flavocytochrome b558. PMN activation stimulates phosphorylation of the cytosolic subunits of NADPH oxidase. Translocation to the membrane forms the active oxidase on the plasma membrane [reviewed in 26]. We investigated whether a reduced NADPH oxidase (gp91 ${ }^{\text {phox }}$ ) surface expression could account for the dampened production of ROS observed in PMNs after CLP. Surprisingly, unstimulated PMNs from CLP mice had somewhat higher surface levels of gp91 ${ }^{\text {phox }}$ compared to control PMNs (fig. 3d), suggesting that a limited activation of PMNs occurred after CLP. For control (non-CLP) PMNs, treatment with C5a or PMA enhanced the surface gp91 $1^{\text {phox }}$ expression (fig. 3d). However, PMA treatment did not significantly increase the gp91 ${ }^{\text {phox }}$ surface expression for CLP PMNs $(\mathrm{p}=0.16)$. The gp91 ${ }^{\text {phox }}$ surface expression on PMA-stimulated CLP PMNs was $30 \%$ lower than on PMA-stimulated control PMNs (fig. 3d). C5a-treated CLP PMNs had a modestly lower surface expression of gp91 ${ }^{\text {phox }}$ compared to C5a-treated control PMNs, but this difference did not reach statistical significance (fig. 3d). Therefore, the lower surface expression of NADPH oxidase may partially explain the reduced ROS generation by bone marrow PMNs following CLP.

To determine the potential contribution of changes in C5aR expression levels on PMNs during CLP, we measured $\mathrm{C} 5 \mathrm{aR}$ on bone marrow PMN populations. Surprisingly, the C5aR surface expression on PMNs was modestly increased 3 days after CLP (fig. 3e). Therefore, the reduced $\mathrm{PMN}$ activation in response to $\mathrm{C} 5 \mathrm{a}$ in vitro (fig. 3a-d) was not due to a decreased C5aR surface expression. Together, these results suggest that there was a prolonged defect in PMN activation and ROS generation following CLP sepsis.

\section{Prolonged CLP-Induced Alterations in Lung Cytokines} during IgG IC ALI

Alveolar macrophages are critical drivers of ALI via their production of inflammatory cytokines and chemokines. Tumor necrosis factor (TNF), IL-1 $\beta$, and IL-10 are important proinflammatory (TNF and IL-1 $\beta$ ) and antiinflammatory (IL-10) cytokines during innate inflammatory responses. We measured the levels of TNF, IL- $1 \beta$, and IL-10 during IgG IC ALI in BALF from non-CLP mice and mice at different time points following CLP. During IgG IC ALI, the BALF levels of TNF, IL- $1 \beta$, and IL-10 were elevated compared to those of sham ALI controls (fig. 4a-c, compare the sham ALI and non-CLP groups, $\mathrm{p}<0.05$ ).

Prolonged CLP-Induced Innate Immune Suppression
Mice that had undergone CLP had significantly reduced levels of TNF in BALF during IgG IC ALI at almost all time points after CLP (fig. 4a). No alterations in the levels of IL-1 $\beta$ were observed following CLP (fig. $4 \mathrm{~b}$ ), suggesting that ALI did not activate the lung inflammasome under the conditions analyzed. However, the IgG IC ALI model was associated with much lower levels of IL- $1 \beta$ production than other models of experimental ALI [e.g. LPS-induced ALI, unpubl. obs.]. IL-10 was found at significantly higher levels in BALF during IgG IC ALI at different time points following CLP compared to the non-CLP group (fig. 4c). It is important to note that the numbers of lavageable alveolar macrophages were unchanged after CLP (fig. 4d). Therefore, CLP sepsis induced long-term (at least 21 days after CLP) skewing in the cytokine milieu during ALI towards a more anti-inflammatory environment. The extreme sensitivity of IL-10 $0^{-/-}$mice to CLP-induced lethality precluded the use of IL- $10^{-/-}$mice to assess whether IL-10 was responsible for defects in TNF production after the induction of ALI [27].

\section{Endotoxemia Is Not Associated with Suppressed Lung Innate Immune Responses}

Mice were rendered endotoxemic to investigate whether suppressed lung innate inflammatory responses were also present in a different model of sepsis. We used a dose of LPS (10 mg/kg of body weight) that resulted in a mortality rate (30\% after 7 days) similar to that occurring in the CLP model that was used for the above studies (fig. 5a). Endotoxemic (day 3 after endotoxin administration) mice had no detectable lung inflammatory response in the absence of IgG IC (fig. 5b, sham). Deposition of IgG IC elevated the levels of albumin found in BALF for both control and endotoxemic mice (fig. 5b, compare the sham and IgG IC ALI groups, $\mathrm{p}<0.005)$. Interestingly, endotoxemia did not significantly alter the levels of albumin found in BALF during IgG IC ALI (fig. 5b), indicating that endotoxemia did not induce the suppression of lung innate immunity. The numbers of PMNs found in the BALF of endotoxemic mice during IgG IC ALI were significantly elevated compared to the values of control mice (fig. 5c), even though the number of circulating PMNs was unchanged by endotoxemia (fig. $5 \mathrm{~d}$ ). This observation may be due to an enhanced recruitment of PMNs into the lung or a reduced apoptosis of PMNs that have entered the lung. Analysis of PMN function based on CD11b levels revealed that endotoxemic (day 3) PMNs did not have activation defects (fig. 5e) and had only a modest reduction of respiratory burst (fig. 5f). Therefore, unlike in the CLP model, endotoxemia did not induce a 


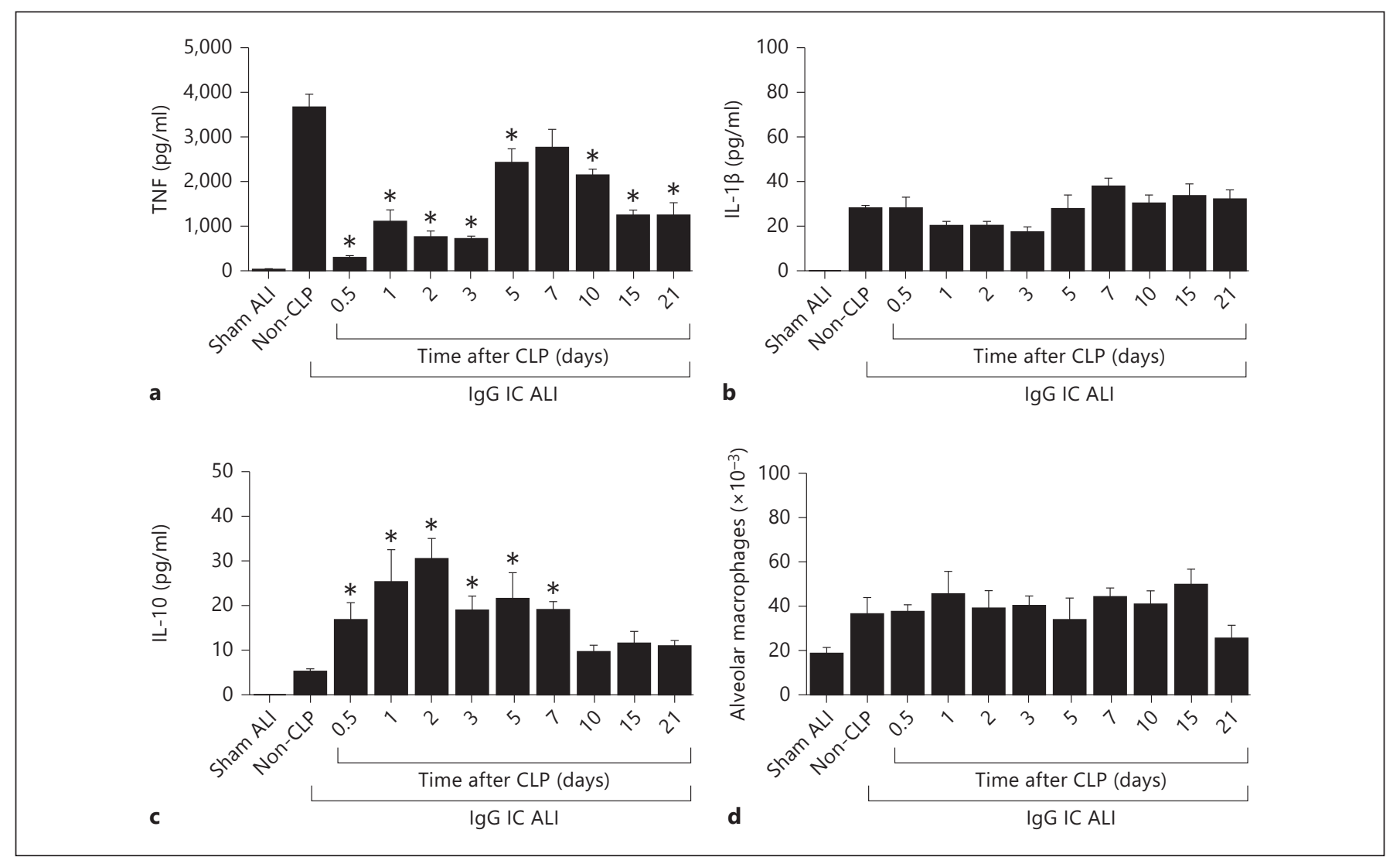

Fig. 4. CLP-induced changes in BALF cytokine levels during IgG IC ALI. BALF was harvested after a two-hit model of CLP and IgG IC ALI at different time points following CLP, as described in figure 1. TNF (a), IL-1 $\beta$ (b), and IL-10 (c) levels in BALF were deter-

significant level of prolonged PMN dysfunction on day 3 after the induction of sepsis.

Similar to the CLP model, the cytokine levels found in BALF during IgG IC ALI were significantly altered by endotoxemia. Specifically, similar to the CLP model, the TNF levels in BALF were reduced by endotoxemia (fig. 5g). Interestingly, the level of IL-1 $\beta$ was enhanced by endotoxemia (fig. 5h), which was not observed in the CLP model and may explain the increased PMN accumulation in this model (fig. 5c) [28]. However, the level of IL-10 found in BALF was enhanced by endotoxemia (fig. 5i), which was similar to the CLP model. Therefore, endotoxemia exerts both similar and distinct effects on cytokine production during IgG IC ALI as in the CLP model.

\section{Prolonged Lymphopenia after CLP or Endotoxemia}

Acute lymphopenia is a known consequence of both CLP and endotoxemia [29]. We observed a prolonged depletion of blood lymphocytes beginning $12 \mathrm{~h}$ after CLP mined by ELISA ( $\mathrm{n} \geq 5$ mice per group). $\mathbf{d}$ The numbers of lavageable alveolar macrophages in BALF samples were determined ( $\mathrm{n}$ $\geq 5$ mice per group). ${ }^{*}$ Differences between the non-CLP and CLP groups were significant, $\mathrm{p}<0.05$. and extending to at least 21 days thereafter (fig. 6a). Similar results were observed after endotoxemia, where lymphopenia extended to at least 15 days after endotoxin administration (fig. 6b). These results suggest that cells of the innate and adaptive immune system are differentially affected during CLP-induced sepsis and endotoxemia.

\section{Discussion}

In this report, we developed and characterized a mouse model that was utilized to investigate persistent sepsis-induced innate immune dysfunction. Previous mouse models of fungal or Gram-negative bacterial infection following CLP have revealed an increased susceptibility to infection following CLP in mice [5-7]. However, the role of acquired immunity during this infection ( $P$. aeruginosa in particular) model is unclear, and a recent report highlighted the role of splenocyte populations in infection pathogenesis 


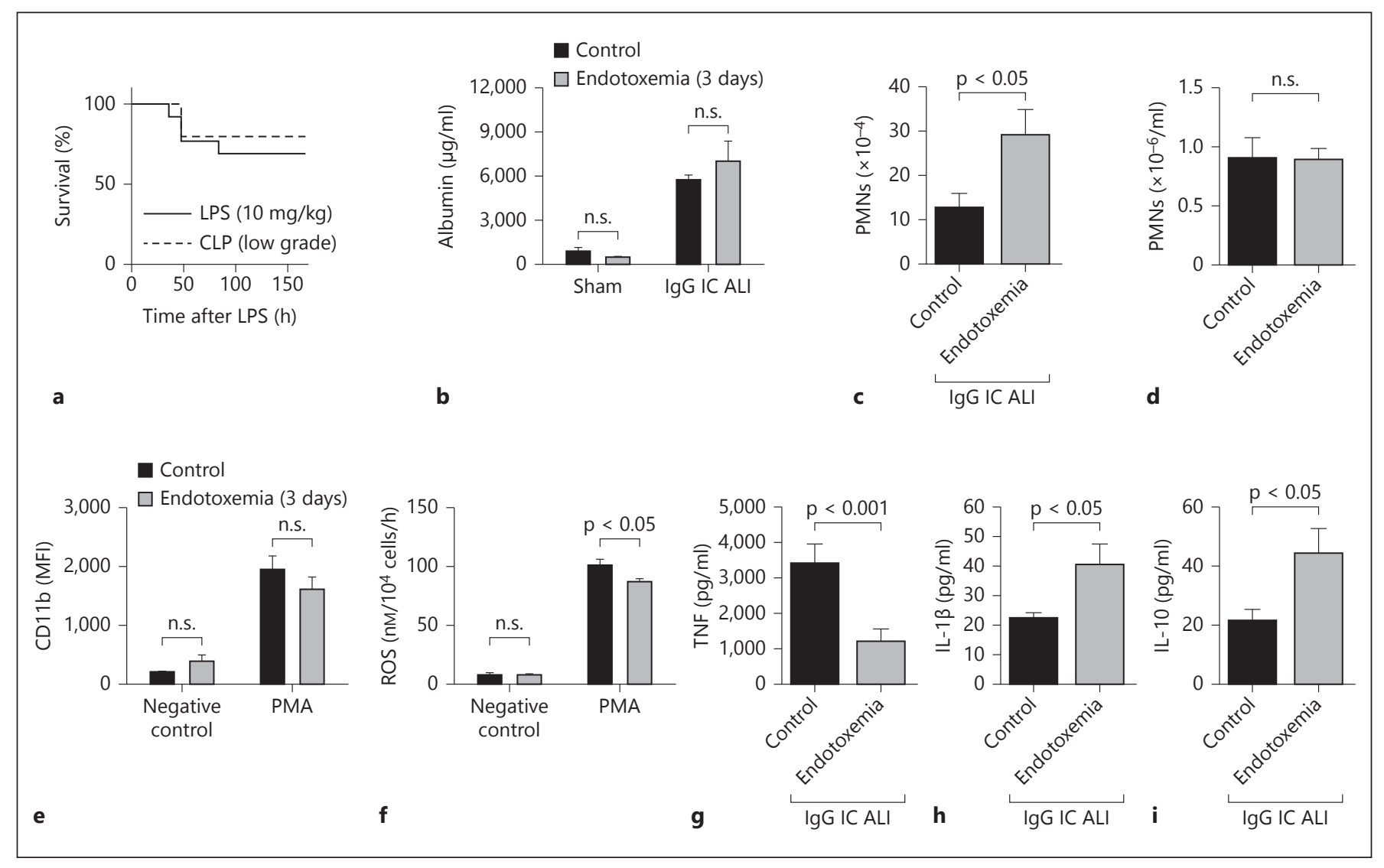

Fig. 5. Endotoxemia ( $10 \mathrm{mg} / \mathrm{kg}$ LPS) does not suppress the lung innate inflammatory response or PMN functions. a Survival of mice following LPS administration $(10 \mathrm{mg} / \mathrm{kg}$, i.p., $\mathrm{n}=13)$ or CLP (low-grade, $\mathrm{n}=10$ ). $\mathbf{b}$ Levels of albumin found in BALF during sham ALI or IgG IC ALI in control and endotoxemic (day 3 ) mice ( $n=6$ mice per group). c Numbers of PMNs in BALF during IgG IC ALI in control and endotoxemic (day 3 ) mice ( $n=6$ mice per group). $\mathbf{d}$ Numbers of PMNs in the blood of control and endotoxemic (day 3 ) mice ( $n=5$ mice per group). e Surface CD11b levels on bone marrow PMNs isolated from control and endotoxemic (day 3) mice following in vitro incubation in the presence or absence of PMA for $1 \mathrm{~h}(\mathrm{n}=4$ mice per group). $\mathbf{f}$ SOD-inhibitable $\mathrm{O}_{2}{ }^{-}$generation by bone marrow $\mathrm{PMNs}$ isolated from control and endotoxemic (day 3 ) mice treated as described in $\mathbf{e}(\mathrm{n}=4$ mice per group). Levels of TNF (g), IL-1 $\beta$ (h), and IL-10 (i) in BALF during IgG IC ALI in control and endotoxemic (day 3$)$ mice ( $n=6$ mice per group). n.s. = Not significant.
Fig. 6. Prolonged depletion of blood lymphocytes after CLP and endotoxemia. The numbers of blood lymphocytes were determined at different time points following CLP-induced sepsis (a) or endotoxemia (b) $(10 \mathrm{mg} / \mathrm{kg}$ LPS) ( $\mathrm{n} \geq 4$ mice per group). * Differences between the control and sepsis groups were significant, $\mathrm{p}<0.05$.

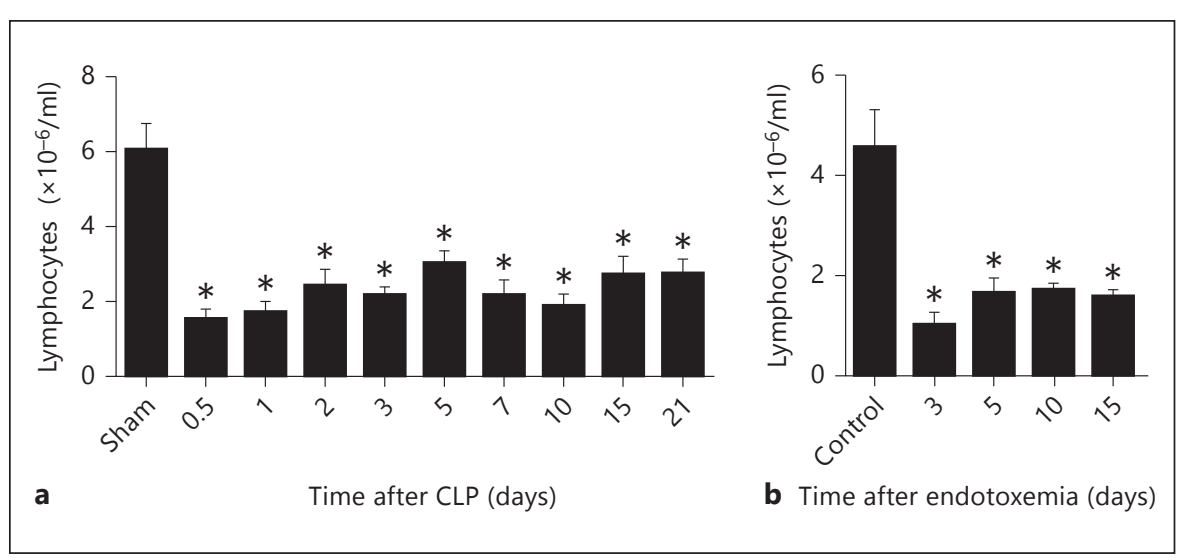

Prolonged CLP-Induced Innate Immune Suppression
J Innate Immun 2014;6:695-705 DOI: $10.1159 / 000362554$ 
following CLP [14]. Therefore, it is difficult to interpret the roles of innate and acquired immunity in this setting. Since the IgG IC ALI model is entirely dependent on innate immune cells (PMNs and alveolar macrophages), it is an appropriate model to investigate innate immune dysfunction following CLP. Furthermore, since opportunistic infections are commonly found in the lung, analyzing the innate immune response in this tissue is clinically relevant.

Previous reports investigating sepsis-induced immunosuppression have examined relatively early time points following the induction of sepsis. To our knowledge, the latest time point examined in previous studies is 7 days following CLP-induced sepsis [11]. However, we now describe impaired innate inflammatory responses extending to weeks after the onset of CLP sepsis. While the severity of impairment at these later times may not necessarily lead to life-threatening illness, it may contribute to the inability to clear acute infections and could lead to persistent health complications like the types observed in the weeks and months after sepsis in humans [4]. Inflammatory suppression following CLP likely represents the innate immune arm of the compensatory anti-inflammatory response syndrome (CARS), which describes a profound impairment of the immune system following the initial cytokine storm of the systemic inflammatory response syndrome (SIRS) in humans. Such impairment is known to render the host susceptible to opportunistic infections or an inability to clear the primary infection [30]. Understanding the effects of CARS on inflammatory cells is essential to developing novel targets for drug intervention.

The differences observed between the CLP and endotoxemia models were striking. It is important to note that endotoxemia was only studied at one time point, and other time points may have resulted in an observation of inflammatory suppression. However, the determination that both models exerted effects on lung cytokine production after 3 days and both resulted in persistent lymphopenia but only CLP induced PMN dysfunction suggests multiple pathways of immune system dysfunction. Furthermore, the preservation of the intensity of IgG IC following endotoxemia (fig. 5b), even though the cytokine milieu was skewed towards a more anti-inflammatory environment (fig. $5 g$, i), suggests that alveolar macrophage dysfunction may not be the predominant force driving the suppression of innate immunity following CLP. Instead, these results suggest that PMN dysfunction may be the primary reason for a reduced innate immune function following CLP. PMN-derived ROS is an important antimicrobial, as can be observed in patients with chronic granulomatous disease, in which phagocytes fail to produce su- peroxide and hydrogen peroxide due to defects in NADPH oxidase [31]. These patients are highly susceptible to infection. The loss of NADPH oxidase expression and activity that we observed following sepsis may be one cellular/ molecular mechanism of CARS and likely leaves the host susceptible to infection. We observed that ALI severity was restored on day 7 after CLP (fig. 1d). However, this result may be due to the huge influx of PMNs into the lung at this time point (fig. 2a). Therefore, PMN function (ROS generation, etc.) is likely to be compromised even at this time point. It will be important to investigate the duration of PMN dysfunction following sepsis.

We have observed prolonged inflammatory suppression after CLP sepsis, which mirrors what has been observed in humans with sepsis. However, whether this phenomenon applies to severe illnesses of other etiologies (e.g. severe trauma) is not yet clear. Studies in humans have demonstrated a prolonged inflammatory state, with the absence of innate immune defects following severe trauma [32-34]. Therefore, etiological differences in SIRS (e.g. sepsis vs. trauma) may impact whether an innate inflammatory dysfunction develops in humans.

In summary, we described prolonged (at least 3 weeks) suppression of the lung innate inflammatory response following CLP-induced sepsis. CLP-induced changes in circulating PMN numbers could explain the reduced lung inflammation at early time points ( $\leq 24 \mathrm{~h}$ ) following CLP. However, later time points, in which PMN numbers were restored or even enhanced above the values of non-CLP mice, displayed a reduced lung inflammation during IgG IC ALI. Reduced inflammatory responses were associated with defects in both PMN and alveolar macrophage function following CLP. Together, these results suggest that in humans long-term innate immune dysfunction may be clinically relevant and its contributions to the increased morbidity and mortality observed following recovery from sepsis deserve further investigation.

\section{Acknowledgements}

The authors thank Beverly Schumann and Sue Scott for their excellent assistance in the preparation of this manuscript. This work was supported by grants from the National Institutes of Health (GM-29507 and GM-61656 to P.A.W. and NHLBI-T32HL007517-29 to J.J.G.).

\section{Disclosure Statement}

The authors declare that they have no financial conflicts of interest. 


\section{References}

$>1$ Angus DC, Linde-Zwirble WT, Lidicker J, Clermont G, Carcillo J, Pinsky MR: Epidemiology of severe sepsis in the united states: analysis of incidence, outcome, and associated costs of care. Crit Care Med 2001;29:13031310.

2 Cohen J: The immunopathogenesis of sepsis. Nature 2002;420:885-891.

$\checkmark 3$ Hotchkiss RS, Karl IE: The pathophysiology and treatment of sepsis. N Engl J Med 2003; 348:138-150.

$>4$ Winters BD, Eberlein M, Leung J, Needham DM, Pronovost PJ, Sevransky JE: Long-term mortality and quality of life in sepsis: a systematic review. Crit Care Med 2010;38:12761283.

$\checkmark 5$ Benjamim CF, Hogaboam CM, Kunkel SL: The chronic consequences of severe sepsis. J Leukoc Biol 2004;75:408-412.

$\checkmark 6$ Benjamim CF, Lundy SK, Lukacs NW, Hogaboam CM, Kunkel SL: Reversal of long-term sepsis-induced immunosuppression by dendritic cells. Blood 2005;105:3588-3595.

7 Deng JC, Cheng G, Newstead MW, Zeng X, Kobayashi K, Flavell RA, Standiford TJ: Sepsis-induced suppression of lung innate immunity is mediated by IRAK-M. J Clin Invest 2006;116:2532-2542.

$>8$ Huber-Lang MS, Younkin EM, Sarma JV, McGuire SR, Lu KT, Guo RF, Padgaonkar VA, Curnutte JT, Erickson R, Ward PA: Complement-induced impairment of innate immunity during sepsis. J Immunol 2002;169: 3223-3231.

$>9$ Huber-Lang MS, Riedemann NC, Sarma JV, Younkin EM, McGuire SR, Laudes IJ, Lu KT, Guo RF, Neff TA, Padgaonkar VA, Lambris JD, Spruce L, Mastellos D, Zetoune FS, Ward PA: Protection of innate immunity by $\mathrm{C} 5 \mathrm{aR}$ antagonist in septic mice. FASEB J 2002;16: 1567-1574.

$>10$ Riedemann NC, Guo RF, Bernacki KD, Reuben JS, Laudes IJ, Neff TA, Gao H, Speyer C, Sarma JV, Zetoune FS, Ward PA: Regulation by $\mathrm{C} 5 \mathrm{a}$ of neutrophil activation during sepsis. Immunity 2003;19:193-202.

-11 Delano MJ, Thayer T, Gabrilovich S, KellyScumpia KM, Winfield RD, Scumpia PO, Cuenca AG, Warner E, Wallet SM, Wallet MA, O’Malley KA, Ramphal R, Clare-Salzer M, Efron PA, Mathews CE, Moldawer LL: Sepsis induces early alterations in innate immunity that impacts mortality to secondary infection. J Immunol 2011;186:195-202.

$>12$ Livingston DH, Appel SH, Wellhausen SR, Sonnenfeld G, Polk HC Jr: Depressed interferon gamma production and monocyte HLA-DR expression after severe injury. Arch Surg 1988;123:1309-1312.
13 Scumpia PO, Delano MJ, Kelly-Scumpia KM, Weinstein JS, Wynn JL, Winfield RD, Xia C, Chung CS, Ayala A, Atkinson MA, Reeves WH, Clare-Salzer MJ, Moldawer LL: Treatment with GITR agonist antibody corrects adaptive immune dysfunction in sepsis. Blood 2007;110:3673-3681.

14 Muenzer JT, Davis CG, Chang K, Schmidt RE, Dunne WM, Coopersmith CM, Hotchkiss RS: Characterization and modulation of the immunosuppressive phase of sepsis. Infect Immun 2010;78:1582-1592.

15 Matthay MA, Ware LB, Zimmerman GA: The acute respiratory distress syndrome. J Clin Invest 2012;122:2731-2740.

16 Gao H, Neff T, Ward PA: Regulation of lung inflammation in the model of igg immunecomplex injury. Annu Rev Pathol 2006;1: 215-242.

17 Rittirsch D, Huber-Lang MS, Flierl MA, Ward PA: Immunodesign of experimental sepsis by cecal ligation and puncture. Nat Protoc 2009;4:31-36.

18 Bosmann M, Sarma JV, Atefi G, Zetoune FS, Ward PA: Evidence for anti-inflammatory effects of C5a on the innate IL-17a/L-23 axis. FASEB J 2012;26:1640-1651.

19 Mei SH, Haitsma JJ, Dos Santos CC, Deng Y, Lai PF, Slutsky AS, Liles WC, Stewart DJ: Mesenchymal stem cells reduce inflammation while enhancing bacterial clearance and improving survival in sepsis. Am J Respir Crit Care Med 2010;182:1047-1057.

20 Fei D, Meng X, Kang K, Nan C, Zhao M, Pan S, Gao M, Yang S, Zhao M: Heme oxygenase-1 modulates thrombomodulin and activated protein $C$ levels to attenuate lung injury in cecal ligation and puncture-induced acute lung injury mice. Exp Lung Res 2012;38:173182.

21 Ulich TR, del Castillo J, McNiece IK, Yi ES, Alzona CP, Yin SM, Zsebo KM: Stem cell factor in combination with granulocyte colonystimulating factor (CSF) or granulocyte-macrophage CSF synergistically increases granulopoiesis in vivo. Blood 1991;78:1954-1962.

22 Lieschke GJ, Grail D, Hodgson G, Metcalf D, Stanley E, Cheers C, Fowler KJ, Basu S, Zhan YF, Dunn AR: Mice lacking granulocyte colony-stimulating factor have chronic neutropenia, granulocyte and macrophage progenitor cell deficiency, and impaired neutrophil mobilization. Blood 1994;84:1737-1746.

23 Zhang P, Quinton LJ, Gamble L, Bagby GJ, Summer WR, Nelson S: The granulopoietic cytokine response and enhancement of granulopoiesis in mice during endotoxemia. Shock 2005;23:344-352.

24 Lindbom L: Regulation of vascular permeability by neutrophils in acute inflammation. Chem Immunol Allergy 2003;83:146-166.
25 Boueiz A, Hassoun PM: Regulation of endothelial barrier function by reactive oxygen and nitrogen species. Microvasc Res 2009;77:2634.

26 Bedard K, Krause KH: The NOX family of ROS-generating NADPH oxidases: physiology and pathophysiology. Physiol Rev 2007;87: 245-313.

27 Latifi SQ, O'Riordan MA, Levine AD: Interleukin-10 controls the onset of irreversible septic shock. Infect Immun 2002;70:44414446.

28 Shanley TP, Peters JL, Jones ML, Chensue SW, Kunkel SL, Ward PA: Regulatory effects of endogenous interleukin-1 receptor antagonist protein in immunoglobulin $\mathrm{G}$ immune complex-induced lung injury. J Clin Invest 1996;97:963-970.

29 Remick DG, Newcomb DE, Bolgos GL, Call DR: Comparison of the mortality and inflammatory response of two models of sepsis: lipopolysaccharide vs. cecal ligation and puncture. Shock 2000;13:110-116.

30 Ward NS, Casserly B, Ayala A: The compensatory anti-inflammatory response syndrome (CARS) in critically ill patients. Clin Chest Med 2008;29:617-628.

31 Song E, Jaishankar GB, Saleh H, Jithpratuck W, Sahni R, Krishnaswamy G: Chronic granulomatous disease: a review of the infectious and inflammatory complications. Clin $\mathrm{Mol}$ Allergy 2011;9:10.

-32 Vanzant EL, Lopez CM, Ozrazgat-Baslanti T, Ungaro R, Davis R, Cuenca AG, Gentile LF, Nacionales DC, Cuenca AL, Bihorac A, Leeuwenburgh C, Lanz J, Baker HV, McKinley B, Moldawer LL, Moore FA, Efron PA: Persistent inflammation, immunosuppression, and catabolism syndrome after sever blunt trauma. J Trauma Acute Care Surg 2014;76:2129.

33 Xiao W, Mindrinos MN, Seok J, Cuschieri J, Cuenca AG, Gao H, Hayden DL, Hennessy L, Moore EE, Minei JP, Bankey PE, Johnson JL, Sperry J, Nathens AB, Billiar TR, West MA, Brownstein $\mathrm{BH}$, Mason $\mathrm{PH}$, Baker $\mathrm{HV}$, Finnerty CC, Jeschke MG, Lopez MC, Klein MB, Gamelli RL, Gibrans NS, Arnoldo B, Xu W, Zhang Y, Calvano SE, McDonald-Smith GP, Schoenfeld DA, Storey JD, Cobb JP, Warren HS, Moldawer LL, Herndon DN, Lowry SF, Maier RV, Davis RW, Tompkins RG; Inflammation and Host Response to Injury Large-Scale Collaborative Research Program: A genomic storm in critically injured humans. J Exp Med 2011;208:2581-2590.

34 Liao Y, Liu P, Guo F, Zhang ZY, Zhang Z: Oxidative burst of circulating neutrophils following traumatic brain injury in human. PLoS One 2013;8:e68963. 